\title{
Un programa de formación en lectura crítica en internet para jóvenes con discapacidad intelectual
}

\section{A training program on critical reading on the internet for young people with intellectual disability}

\section{Palabras clave}

Lectura crítica, alfabetización digital, programa de formación, discapacidad intelectual.

\section{Keywords}

Critical reading, digital literacy, training program, intellectual disability.

\author{
Pablo Delgado Herrera \\ <pablo.delgado@uv.es>
}

Universidad de Valencia. España

\section{Vicenta Ávila Clemente <vicenta.avila@uv.es>}

Universidad de Valencia. España

Inmaculada Fajardo Bravo <inmaculada.fajardo@uv.es>

Universidad de Valencia. España

Ladislao Salmerón González <ladislao.salmeron@uv.es>

Universidad de Valencia. España

\subsection{El contexto}

En la actualidad, internet es el principal medio de información para la mayoría de los ciudadanos, cuyo uso por parte de niños y jóvenes está muy generalizado. Igualmente, el uso por parte de las personas con discapacidad intelectual (DI) se ha incrementado en los últimos años, generando un debate sobre sus beneficios y riesgos (Chiner et al., 2017). Por un lado, internet permite reducir e incluso eliminar algunas de las barreras que limitan el acceso de las personas con DI a ciertas actividades cotidianas, especialmente aquellas barreras provocadas por los prejuicios y actitudes de los demás. Por tanto, su uso aumenta las posibilidades de dicha población para participar activamente en la sociedad, lo que ofrece importantes

\footnotetext{
1. Agradecemos a los estudiantes y centros participantes por su colaboración desinteresada en el desarrollo del proyecto, y a Guillermo Zapata por su colaboración en la evaluación. Esta investigación ha sido financiada por un proyecto de I+D del Ministerio de Economía y Competitividad (Secretaría General de Universidades), con referencia EDU2014-59422P. Asociada a dicho proyecto, la actividad investigadora del primer autor ha sido financiada por la convocatoria de ayudas para contratos pre-doctorales del Ministerio de Economía y Competitividad, con referencia BES-2015-076055 y cofinanciada por el Fondo Social Europeo. El Programa de Lectura Crítica en internet para Jóvenes con Discapacidad Intelectual está disponible para su uso con fines académicos, bajo petición a ladislao.salmeron@uv.es.
}

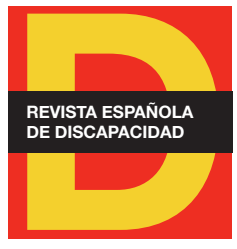

Para citar:

Delgado, P. et al. (2018): "Un programa de formación en lectura crítica en internet para jóvenes con discapacidad intelectual". Revista Española de Discapacidad, 6 (II): 229-245.

Doi: <https://doi.org/10.5569/23405104.06.02.13> 
beneficios relacionados con las oportunidades de vida (Chadwick et al., 2013). Sin embargo, los programas dirigidos a la formación de esta población se han centrado tradicionalmente en habilidades académicas, como la comprensión lectora o las matemáticas; en habilidades de la vida independiente, como la autonomía personal, y en habilidades ocupacionales. Aunque es innegable la necesidad de las anteriores competencias, creemos oportuno ir más allá y ofrecer una formación en aquellas habilidades básicas que permitan a las personas con DI desenvolverse con éxito en la actual realidad digital de la era de la información. El propósito es ampliar el abanico de oportunidades hacia la autodeterminación, definida como la capacidad de actuar "como el principal agente causal en la propia vida y tomar decisiones con respecto a la calidad de vida, libre de influencias o interferencias externas indebidas" (Wehmeyer, 1996: 22). Sin embargo, no podemos soslayar que quienes son vulnerables en la vida cotidiana tienden a serlo también en internet (Mitchell et al. , 2011). En este sentido, las personas con DI han de hacer frente a algunas dificultades cognitivas que hacen que el uso de internet sea un reto para ellas. Dos de las más importantes son su bajo nivel de habilidades lectoras (Fajardo et al., 2014) y su alto nivel de credulidad (Greenspan et al., 2001). Estas limitaciones contribuyen a que las personas con DI tiendan a acceder y aceptar como válida información poco fiable de internet (Salmerón et al., en prensa; Salmerón et al., 2016). Así, de acuerdo con recientes trabajos de investigación que proponen la necesidad de programas de alfabetización compleja para personas con DI (Lundberg y Reichenberg, 2013), el programa de intervención que presentamos se configura como una herramienta dirigida al entrenamiento de personas con DI en algunas de las habilidades básicas necesarias para evaluar críticamente la fiabilidad de la información disponible en internet.

\subsection{Los inicios}

Las anteriores cuestiones convergen en el intercambio de experiencias facilitado por las 'IX Jornadas Científicas Internacionales de Investigación sobre Personas con Discapacidad', celebradas en 2015 en Salamanca, donde se presentó la experiencia del Club de Lectura Fácil para jóvenes con DI desarrollada por la Asociación de Familiares de Personas con Discapacidad Intelectual Afanias Madrid (Zarcero, 2015). Con inspiración en este proyecto, planteamos la posibilidad de poner en marcha un Club de Lectura Crítica de la información disponible en internet, que se concretó en una experiencia piloto en colaboración con dicha asociación. Para su diseño nos apoyamos en la propuesta desarrollada por el proyecto MD-Skills, de la Agencia Nacional Francesa de Investigación ${ }^{2}$ y la Fundación Alemana de Investigación ${ }^{3}$. Centrado en la formación en lectura crítica de múltiples textos en adolescentes con desarrollo normativo, este proyecto se consolida como fruto de la colaboración internacional entre los grupos de investigación liderados por los investigadores Jean-François Rouet (Universidad de Poitiers, Francia) y Marc Stadtler (Universidad de Bochum, Alemania).

Como resultado de la aplicación piloto, tanto los participantes (once adultos jóvenes con DI) como los educadores de la asociación Afanias (quienes aplicaron las sesiones de formación) indicaron sentirse satisfechos con el desarrollo del programa. Asimismo, obtuvimos resultados positivos en relación con el aprendizaje de los participantes (Fajardo et al., 2016). Tras la retroalimentación recibida por esta experiencia, se mejoró el diseño del programa y se aplicó a un total de 33 jóvenes estudiantes en la asociación Asindown y en las Escuelas San José-Jesuitas, ambas instituciones situadas en la ciudad de Valencia. Con una edad

2. Más información: http://www.agence-nationale-recherche.fr/Project-ANR-12-FRAL-0015.

3. Más información: http://gepris.dfg.de/gepris/projekt/233518543. 
media de 19 años y una DI moderada o leve (CI medio igual a 52.32), sus habilidades lectoras correspondían a los tres primeros cursos de Educación Primaria. Además, el $94 \%$ de dichos participantes declaró usar internet de manera regular. El programa ayudó a los participantes a tener en cuenta las fuentes de información en internet y mejoró sus habilidades para evaluar su fiabilidad (Delgado et al., en prensa). Por todo ello, creemos oportuno presentarlo en este artículo, ofreciendo a los profesionales del ámbito educativo una herramienta de formación para la mejora de la competencia digital en personas con DI.

\section{El programa}

\subsection{Objetivos generales}

El Marco Europeo sobre la Competencia Digital para la Ciudadanía (DigComp 2.0) (Vuorikari et al., 2016) establece la habilidad para evaluar críticamente la credibilidad y la fiabilidad de las fuentes de información digitales como parte esencial de la competencia digital. De esta forma, el programa que presentamos trata de sensibilizar a los participantes acerca de la habitual existencia de información contradictoria en internet sobre un mismo tema, y, principalmente, enseñarles a identificar y evaluar tres factores básicos relacionados con la fiabilidad y la validez de dicha información: (1) El control editorial de la fuente, (2) el conocimiento acreditado por la profesión del autor y (3) la intención del autor o del sitio web.

\subsection{Fases del programa y objetivos específicos}

El programa de intervención consta de cuatro fases: 1) Pre-test, una sesión de evaluación previa a la intervención, 2) fase de intervención-instrucción, durante un total de cuatro sesiones, 3) fase intervenciónpráctica, con tres sesiones y 4) post-test, una sesión de evaluación posterior a la intervención. De esta forma, queda constituido por siete sesiones de intervención y dos sesiones de evaluación, de una duración aproximada de 45-60 minutos. A continuación, se presenta una descripción de cada fase:

1. Evaluación pre-test: Se presenta a los participantes una situación en la que un personaje necesita ayuda para decidir, entre dos posibilidades, qué web es más recomendable para aprender acerca de un tema. Los participantes deben elegir en qué página es oportuno confiar y justificar su respuesta.

2. Fase de intervención-instrucción: Compuesta por cuatro módulos cada uno de los cuales se centra en uno de los cuatro factores relacionados con la fiabilidad de las fuentes de información que son objetivos de aprendizaje del programa. Cada módulo es impartido en una única sesión:

- Módulo 1: ¿Qué dice internet? Objetivos: (1) Identificar que en internet habitualmente existe información contradictoria sobre un mismo tema y (2) introducir el concepto de lectura crítica: hay que decidir en qué información es posible confiar.

- Módulo 2: ¿Dónde se encuentra la información en internet? Objetivos: (1) Conocer la existencia de diferentes tipos de páginas web (blogs, periódicos, empresas, instituciones públicas...), (2) conocer que existen webs en las que no existe control editorial y puede publicar cualquier persona (foros, 
blogs personales, redes sociales...), mientras que otras mantienen un control editorial sobre la información que se publica (periódicos, instituciones...) y (3) identificar aquellas páginas en las que los contenidos están controlados como criterio de fiabilidad.

- Módulo 3: ¿Quién ofrece la información en internet? Objetivos: (1) Introducir la importancia de atender al autor o transmisor de la información y (2) identificar el conocimiento del autor respecto al contenido de la información como criterio de fiabilidad, teniendo en cuenta su profesión y su experiencia.

- Módulo 4: ¿Qué intención tiene la fuente de información? Objetivos: (1) Introducir el concepto de intención de la página web o del autor del contenido, (2) sensibilizar sobre la importancia de identificar dicha intención como criterio de fiabilidad (informativa, comercial, ayuda...) y (3) identificar y valorar la interacción entre la experiencia del autor y la intención de la página web (ejemplo: un autor puede ser experto, pero transmitir la información en una página con intenciones comerciales).

3. Fase de intervención-práctica (Módulos 5 al 7): Su objetivo es enseñar a los participantes a valorar la fiabilidad de la información teniendo en cuenta de manera global todos los criterios trabajados en los módulos anteriores. Se practica esta valoración general a través de la pregunta ‘Cómo explico mi respuesta?', cuya respuesta se construye a su vez con base en las siguientes preguntas, una estructura de trabajo que se mantiene constante a lo largo de los tres módulos de esta fase de intervención-práctica: (1) ¿Qué dice el texto?, (2) ¿Quién lo escribe? y (3) ¿Dónde está escrito?

4. Evaluación post-test: Su procedimiento es idéntico a la sesión de evaluación previa, pero las páginas web empleadas y sus contenidos son distintos, permitiendo así la evaluación de los resultados de aprendizaje del programa.

\subsection{Materiales}

- Presentaciones:

Las sesiones son impartidas a través de una presentación tipo PowerPoint que ayuda a contextualizar los contenidos. Cada una de las presentaciones refleja una pequeña situación ficticia en la que dos personajes buscan información para aprender acerca de un tema de carácter socio-científico y encuentran dos páginas web con información contradictoria entre ellas. En cada módulo de la fase de intervencióninstrucción, los personajes exponen respectivamente uno de los criterios que han de ser tenidos en cuenta para valorar la fiabilidad de la información (ver sección 'Fases del programa y objetivos específicos'), además de cómo valorarlos. Un ejemplo de una de las diapositivas de las presentaciones de estos primeros cuatro módulos puede verse en la Figura 1. 
Figura 1. Ejemplo de una de las diapositivas, en la que un personaje busca información en internet

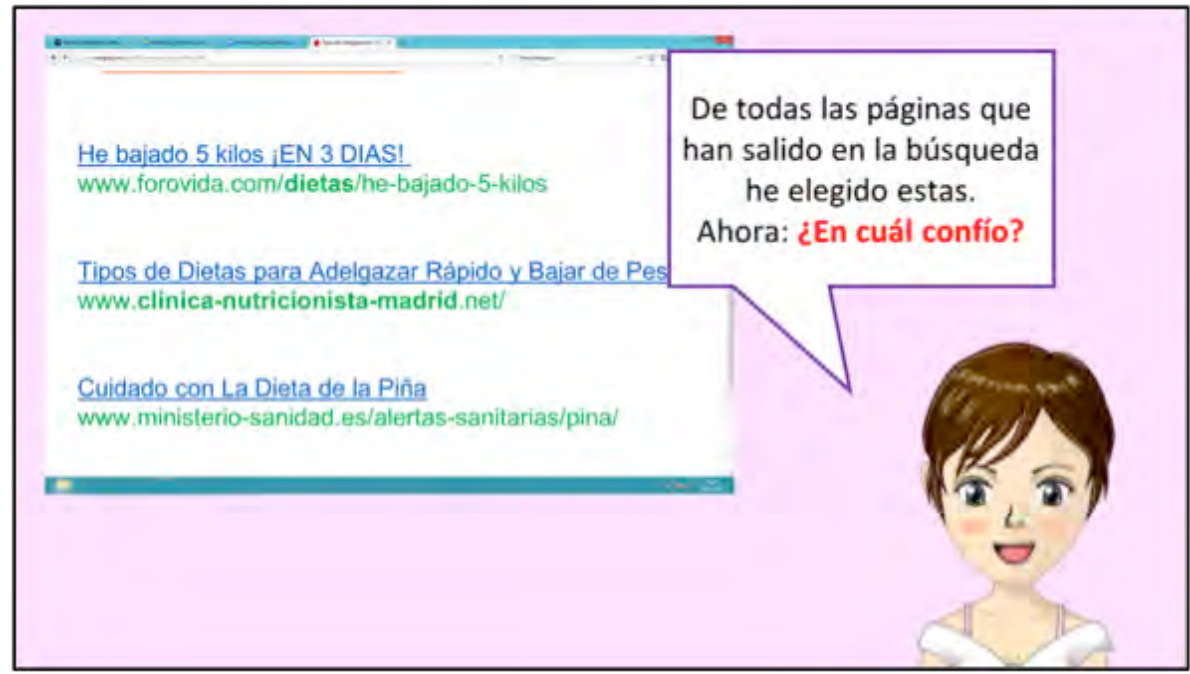

Fuente: elaboración propia.

En los tres módulos de la fase de intervención-práctica, la presentación constituye una historieta algo más compleja. En ella, los personajes, además de buscar información, tratan de evaluar paso a paso la fiabilidad de la información encontrada de nuevo en dos páginas web distintas. Así, mientras uno de ellos tan solo tiene en cuenta el contenido de la información y sus conocimientos previos, el otro atiende, paso a paso, a los distintos criterios de fiabilidad trabajados a lo largo de los cuatro módulos de la fase de instrucción. La diferencia entre el proceder de ambos personajes constituye el material de trabajo para aplicar la metodología de contraste de casos, que se describirá más adelante.

Por último, las presentaciones utilizadas para las sesiones de evaluación de los alumnos (pre y post) son más sencillas. Aquí un personaje presenta dos páginas web que ha encontrado al buscar información y solicita ayuda para decidir en qué página debe confiar.

- Páginas web:

Como ya hemos mencionado, en cada sesión son utilizadas dos páginas web que presentan dos visiones distintas acerca de un mismo tema, ofreciendo por tanto información en conflicto. Además, y más importante para los objetivos del programa, las fuentes de información (web y autor del contenido) difieren en su fiabilidad, la cual depende, tal y como se ha expuesto, del campo profesional del autor (experto vs. no experto en el tema), la intención del sitio web (ejemplo: la web de una compañía comercial vs. la web de una asociación sin ánimo de lucro), y el grado de control sobre la información publicada (ejemplo: un foro web vs. la página de un hospital).

Por tanto, cada sesión se desarrolla con base en un par páginas cuyos contenidos se encuentran en conflicto. Es importante señalar que las temáticas de los contenidos fueron seleccionadas por los par- 
ticipantes del programa piloto de acuerdo con sus propios intereses. Los textos se obtuvieron a partir de páginas web reales y fueron simplificados para su lectura fácil siguiendo las recomendaciones de la Federación Internacional de Asociaciones de Bibliotecas y Bibliotecarios (IFLA) (Nomura et al., 2010). Las páginas web simulan una web real tal y como es visionada en un navegador, con la siguiente información disponible: dirección url, el logo de la organización o la empresa propietaria de la web y el autor de la información (ver Figura 2). En la Tabla 1, se muestran los títulos de los contenidos y las características de las fuentes de información empleadas en cada una de las sesiones.

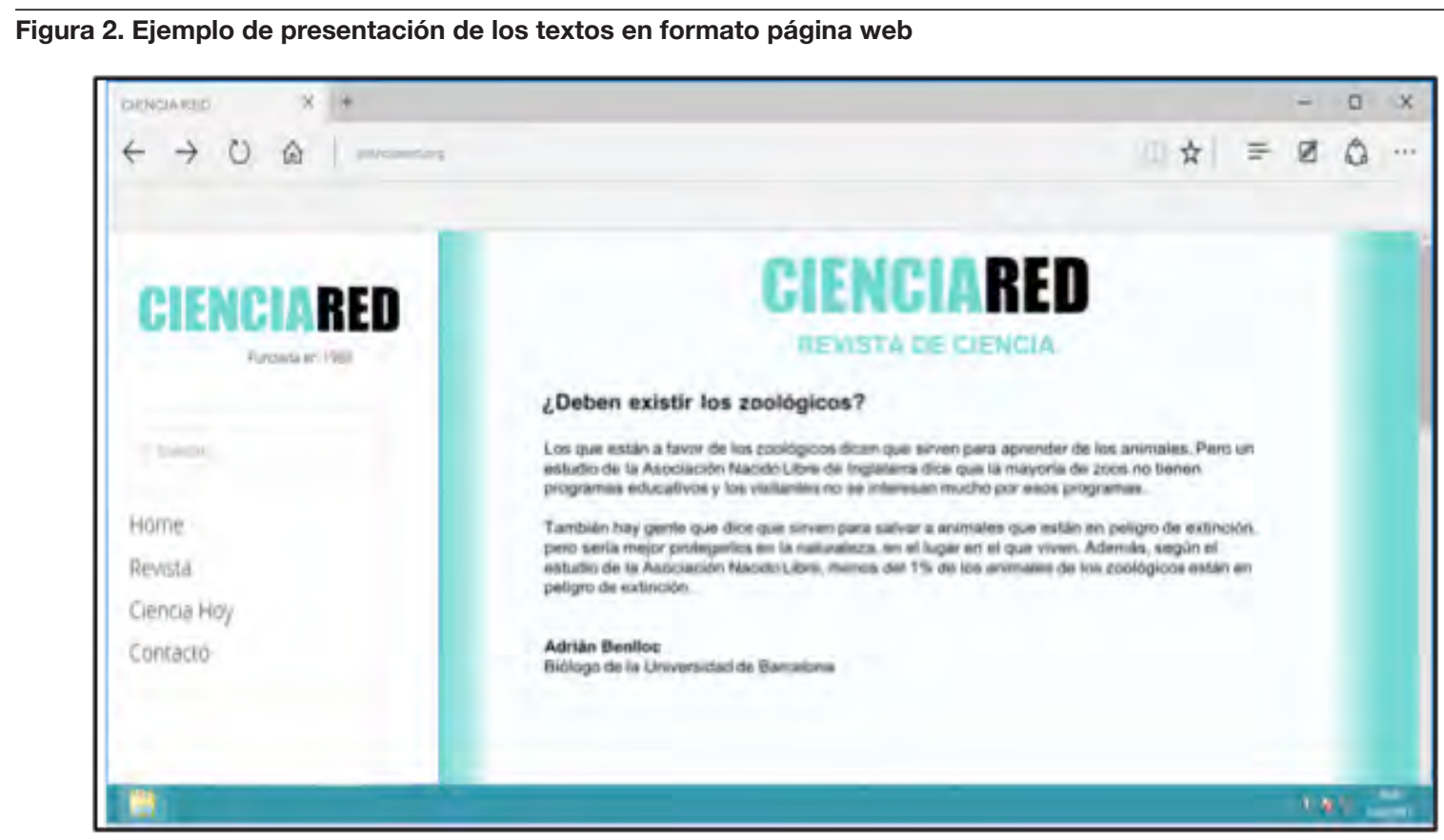




\begin{tabular}{|c|c|c|c|}
\hline Sesiones & Contenidos & Características de la fuente de información & $\begin{array}{l}\text { Núm. de } \\
\text { palabras }\end{array}$ \\
\hline \multirow{2}{*}{ Pre-test } & $\begin{array}{l}\text { Los móviles no son malos para la } \\
\text { vista. }\end{array}$ & $\begin{array}{l}\text { Web: comercial } \\
\text { Autor: jefe de ventas (no experto) } \\
\text { Intención: vender (no benévola) }\end{array}$ & 164 \\
\hline & $\begin{array}{l}\text { La luz de la pantalla de tu móvil } \\
\text { daña tu vista. }\end{array}$ & $\begin{array}{l}\text { Web: periódico } \\
\text { Autor: oftalmólogo (experto) } \\
\text { Intención: informar (benévola) }\end{array}$ & 141 \\
\hline \multirow{2}{*}{ Módulo 1} & Los peligros del dopaje cerebral. & $\begin{array}{l}\text { Web: revista científica } \\
\text { Autor: neurólogo (experto) } \\
\text { Intención: informar (benévola) }\end{array}$ & 115 \\
\hline & $\begin{array}{l}\text { Dopaje cerebral: La solución para } \\
\text { el próximo examen. }\end{array}$ & $\begin{array}{l}\text { Web: comercial } \\
\text { Autor: jefe de marketing (no experto) } \\
\text { Intención: vender (no benévola) }\end{array}$ & 142 \\
\hline Módulo 2 & $\begin{array}{l}\text { El contenido de los textos no es } \\
\text { relevante, solo el tipo de web } \\
\text { donde aparecen. El tema es el } \\
\text { mismo que el del módulo } 1 .\end{array}$ & $\begin{array}{l}\text { Web 1: institución educativa (publicación controlada) } \\
\text { Web 2: periódico (publicación controlada) } \\
\text { Web 3: comercial (publicación controlada) } \\
\text { Web 4: foro (publicación abierta) } \\
\text { Web 5: blog (publicación abierta) }\end{array}$ & - \\
\hline \multirow{2}{*}{ Módulo 3} & $\begin{array}{l}\text { Nuestros datos y los teléfonos } \\
\text { móviles. ¿Qué riesgos existen? }\end{array}$ & $\begin{array}{l}\text { Web: oficina de ayuda al internauta. } \\
\text { Autor: ingeniera informática (experta) } \\
\text { Intención: informar (benévola) }\end{array}$ & 135 \\
\hline & $\begin{array}{l}\text { Los problemas de seguridad en el } \\
\text { móvil están sobrevalorados. }\end{array}$ & $\begin{array}{l}\text { Web: foro } \\
\text { Autor: estudiante de ESO (no experta) } \\
\text { Intención: contar su experiencia (benévola) }\end{array}$ & 160 \\
\hline \multirow{2}{*}{ Módulo 4} & Consumir carne no causa cáncer. & $\begin{array}{l}\text { Web: comercial } \\
\text { Autor: gerente carnicería (experto) } \\
\text { Intención: vender (no benévola) }\end{array}$ & 91 \\
\hline & $\begin{array}{l}\text { Carne procesada: comer con } \\
\text { precaución. }\end{array}$ & $\begin{array}{l}\text { Web: Organización Mundial de la Salud } \\
\text { Autor: medico nutricionista (experto) } \\
\text { Intención: informar (benévola) }\end{array}$ & 130 \\
\hline \multirow{2}{*}{ Módulo 5} & $\begin{array}{l}\text { Bebidas energéticas: peligro para } \\
\text { los jóvenes. }\end{array}$ & $\begin{array}{l}\text { Web: hospital } \\
\text { Autor: médica de familia (experta) Intención: informar } \\
\text { (benévola) }\end{array}$ & 80 \\
\hline & Bebidas energéticas y salud. & $\begin{array}{l}\text { Web: comercial } \\
\text { Autor: nutricionista (experta) } \\
\text { Intención: vender (no benévola) }\end{array}$ & 110 \\
\hline \multirow{2}{*}{ Módulo 6} & ¿A favor o en contra de los zoos?' & $\begin{array}{l}\text { Web: foro } \\
\text { Autor: usuario de foro (experiencia desconocida) } \\
\text { Intención: compartir su opinión (benévola) }\end{array}$ & 116 \\
\hline & ¿Deben existir los zoológicos? & $\begin{array}{l}\text { Web: revista de ciencia } \\
\text { Autor: biólogo (experto) } \\
\text { Intención: informar (benévola) }\end{array}$ & 108 \\
\hline \multirow{2}{*}{ Módulo 7} & $\begin{array}{l}\text { Los beneficios del agua mineral } \\
\text { natural }\end{array}$ & $\begin{array}{l}\text { Web: comercial } \\
\text { Autor: directora comercial (no experta) Intención: vender (no } \\
\text { benévola) }\end{array}$ & \multirow{2}{*}{$\begin{array}{l}\text { Se utilizan } \\
\text { dos } \\
\text { videos }\end{array}$} \\
\hline & $\begin{array}{l}\text { ¿El agua mineral embotellada es } \\
\text { mejor que la del grifo? }\end{array}$ & $\begin{array}{l}\text { Web: asociación de consumidores } \\
\text { Autor: doctora (experta) } \\
\text { Intención: informar (benévola) }\end{array}$ & \\
\hline
\end{tabular}




\begin{tabular}{|l|l|l|c|}
\hline Sesiones & Contenidos & Características de la fuente de información & $\begin{array}{c}\text { Núm. de } \\
\text { palabras }\end{array}$ \\
\hline \multirow{3}{*}{ Post-test } & $\begin{array}{l}\text { Las redes wi-fi no son perjudiciales } \\
\text { para la salud }\end{array}$ & $\begin{array}{l}\text { Web: comercial } \\
\text { Autor: director de publicidad (no experto) } \\
\text { Intención: vender (no benévola) }\end{array}$ & 167 \\
\cline { 2 - 4 } & \multirow{2}{*}{ ¿Son perjudiciales las redes wi-fi? } & $\begin{array}{l}\text { Web: periódico } \\
\text { Autor: doctora (experta) } \\
\text { Intención: informar (benévola) }\end{array}$ & 141 \\
\hline
\end{tabular}

Fuente: elaboración propia.

Adicionalmente, una de las sesiones contiene páginas web que presentan el contenido en formato audiovisual, un medio de presentación de elevado uso en la actualidad con fines informativos o de aprendizaje (ver Figura 3). Estos vídeos presentan a un profesional que expone argumentos sobre el tema ' ¿Es mejor el agua embotellada que la del grifo?'.

\section{Figura 3. Ejemplo de página web cuyo contenido se presenta en formato audiovisual}

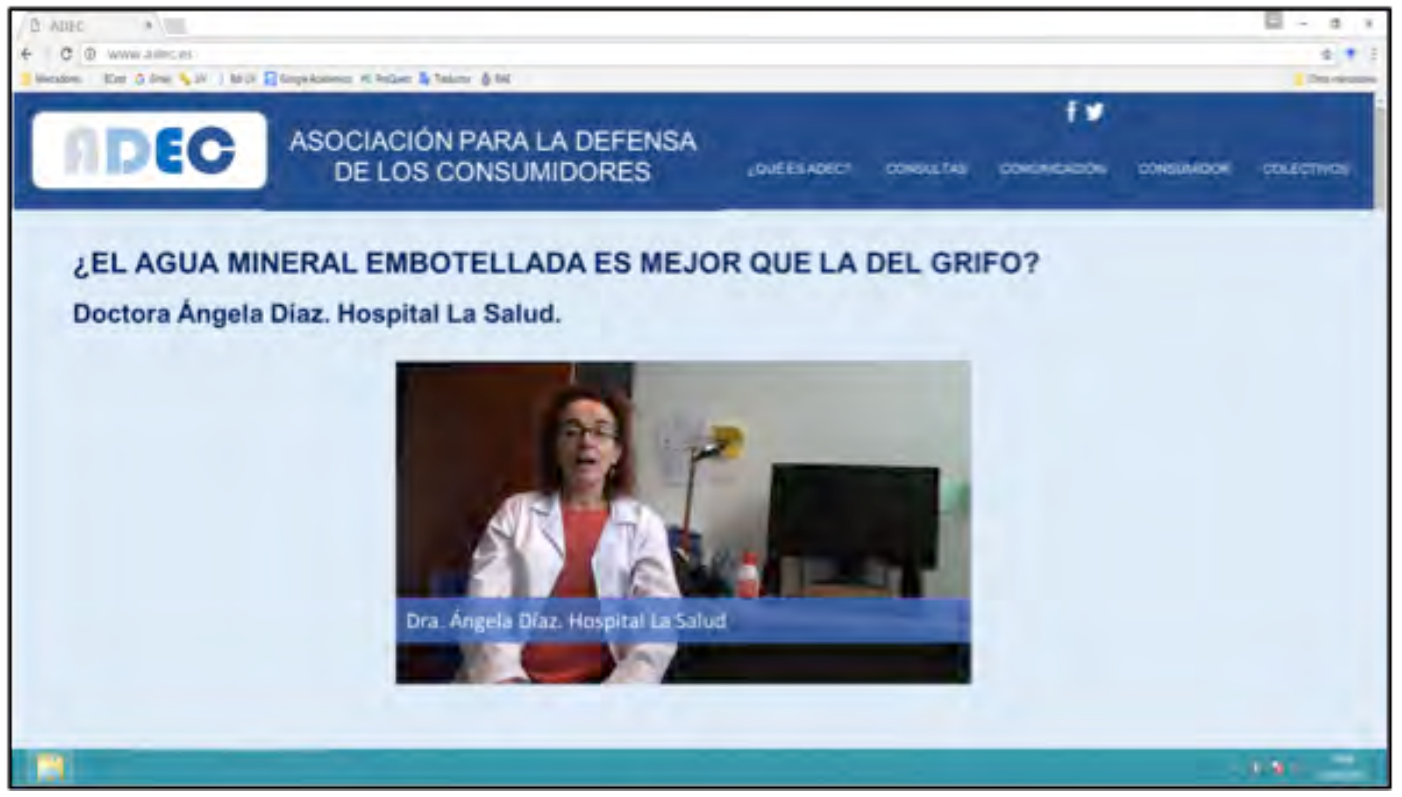

Fuente: elaboración propia.

- Tarjetas de votación:

A lo largo de las distintas sesiones del programa los participantes deben tomar decisiones acerca de la fiabilidad de distintas páginas web. Estas respuestas son emitidas mediante una tarjeta de votación que es levantada por todos los participantes al mismo tiempo, de forma que la decisión no resulte influida 
por la tomada por el resto de compañeros. Existen dos pares distintos de tarjetas. Uno de ellos permite tomar decisiones acerca de la posibilidad de confiar en una web (tarjetas 'sí/no', ver Figura 4), mientras que el otro permite seleccionar la web fiable de entre dos posibilidades (tarjetas '1/2').

\section{Figura 4. Tarjetas de votación 'Sí/No'}

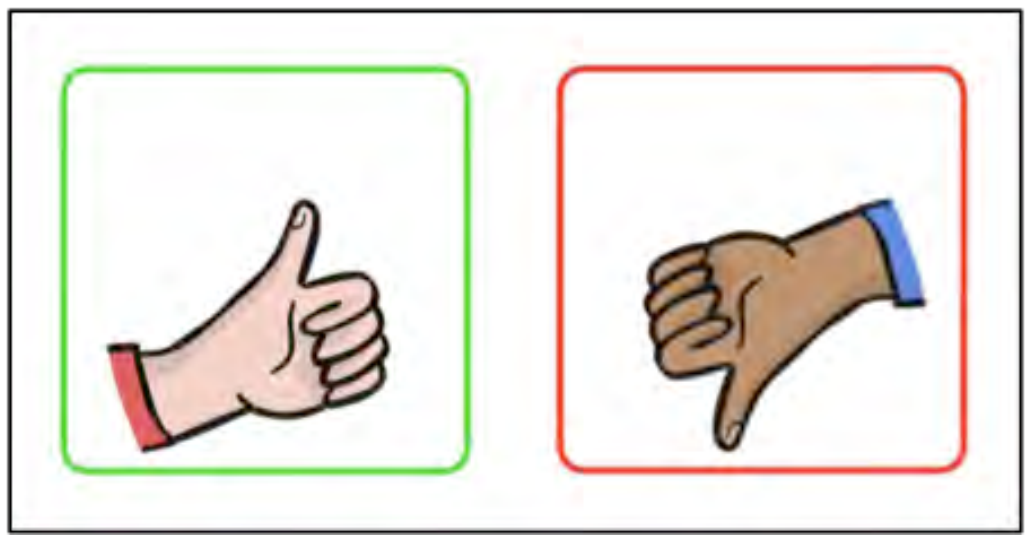

Fuente: elaboración propia.

- $\quad$ Carteles de registro:

La metodología de las sesiones exige la participación de los alumnos. Como se expondrá más adelante, la discusión grupal es una herramienta fundamental. De esta forma, las verbalizaciones y respuestas de los participantes durante las discusiones y actividades pueden ser registradas por el conductor del taller en un cartel colocado en un lugar visible para el grupo y de un tamaño que permita su legibilidad. Su función es doble: por un lado, sirven para apoyar las discusiones manteniendo presente los argumentos aportados y facilitando su recapitulación. Por otro, son de indudable utilidad para valorar la participación de los alumnos y la evolución de su proceso de aprendizaje. Aunque estos carteles pueden construirse ad hoc por los profesionales que deseen aplicar el programa, adaptándose a sus necesidades e incluso planteándose en sí mismo como una actividad para realizar por los alumnos, la Figura 5 es un ejemplo de presentación. 
Figura 5. Ejemplo de presentación de los carteles de registro, con posibles anotaciones

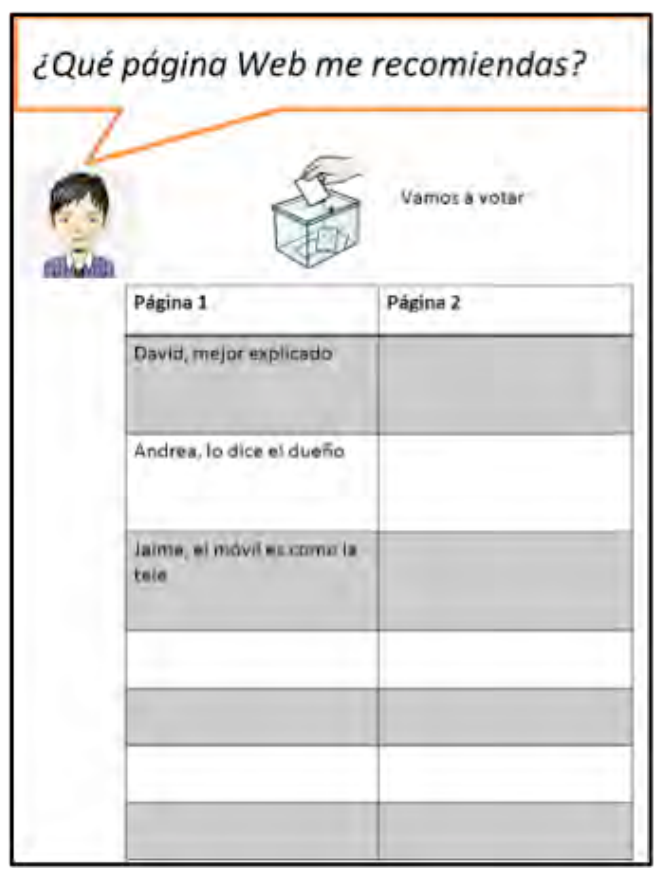

Fuente: elaboración propia.

- Guía de aplicación:

El programa consta de una guía de aplicación en la que se detalla cada sesión, sus objetivos de aprendizaje y cada uno de los pasos del procedimiento de aplicación.

\subsection{Metodología}

En una revisión de estudios de intervención en alfabetización, Copeland y Keefe (2017) concluyen que muchas de las estrategias basadas en prácticas de investigación que son aplicadas en programas para estudiantes con desarrollo normativo son asimismo efectivas para estudiantes con discapacidad intelectual. Con base en estas afirmaciones, nuestro programa fue diseñado partiendo de la adaptación de anteriores trabajos de intervención desarrollados por Stadtler et al. (2016), y sobre marcos teóricos que asumen que la atención a las fuentes de información y la evaluación de su fiabilidad es una actividad crucial cuando hacemos frente a distintos textos cuya información es contradictoria (Bråten et al., 2018).

La metodología instruccional queda conformada por una combinación de distintos métodos adaptados a lectura guiada y grupal, incluyendo instrucción directa, grupos de discusión, modelado y contraste de casos, cuya eficacia ha sido probada a través de estudios de intervención en el desarrollo de la lectura y habilidades sociales en estudiantes con DI (Copeland y Keefe, 2017; Hetzroni y Banin, 2017): 
- Lectura guiada y estructurada en voz alta: dadas las habituales dificultades lectoras de las personas con DI, el programa está diseñado para la lectura en voz alta por parte del instructor de los contenidos, o por alguno de los alumnos si su nivel de lectura lo permite. El instructor dirige la sesión y asegura la comprensión del texto por parte de los alumnos, realizando preguntas de comprensión y aclaraciones a lo largo de la lectura. En el módulo de trabajo en que se utilizan vídeos, el instructor también guía la comprensión de sus contenidos. Aun así, esta circunstancia queda abierta a las decisiones que puedan tomarse respecto a las necesidades de los participantes, pudiendo adaptarse a su nivel de habilidad lectora.

- Modelado: como se ha expuesto con anterioridad, los distintos módulos de formación son conducidos a través de personajes que buscan información e internet y tratan de decidir en cuál deben confiar. De esta forma, las aportaciones de dichos personajes, que son verbalizadas y guiadas por el instructor del programa, se configuran como modelos de actuación para los estudiantes (ver Figura 6).

Figura 6. Ejemplo de diapositiva de modelado.

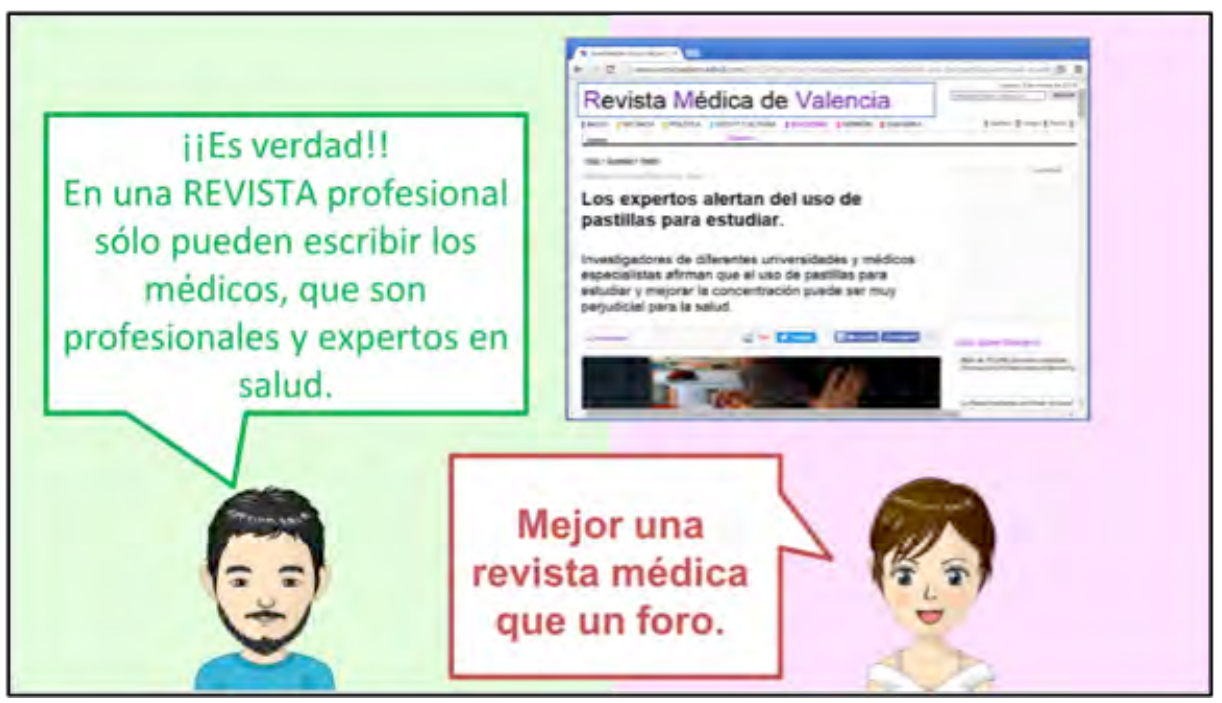

Fuente: elaboración propia.

- Contraste de casos: como ya hemos introducido anteriormente al describir los materiales, durante los módulos de la fase de instrucción-práctica los personajes conductores de las sesiones tratan de evaluar la fiabilidad de la información que encuentran en internet. En este proceso, uno de los personajes basa su decisión en el contenido de la información y en sus conocimientos previos, mientras que el otro construye su argumentación y decisión final con base en los distintos criterios de fiabilidad expuestos como objetivos del programa en apartados anteriores. Esta situación representa el contraste de casos, pues con la guía del instructor, pero apoyándose en los comentarios del grupo, se comparan ambas argumentaciones y se decide cuál de los dos personajes ha seguido los criterios apropiados para decidir acerca de la fiabilidad de la información (Salmerón, 2013). Estos casos de contraste pueden considerarse asimismo como instrucción por modelado, pues aquel personaje que basa sus decisiones en todos 
los criterios de fiabilidad es señalado como un modelo apropiado. En la Figura 7 puede visualizarse un ejemplo de contraste de casos.

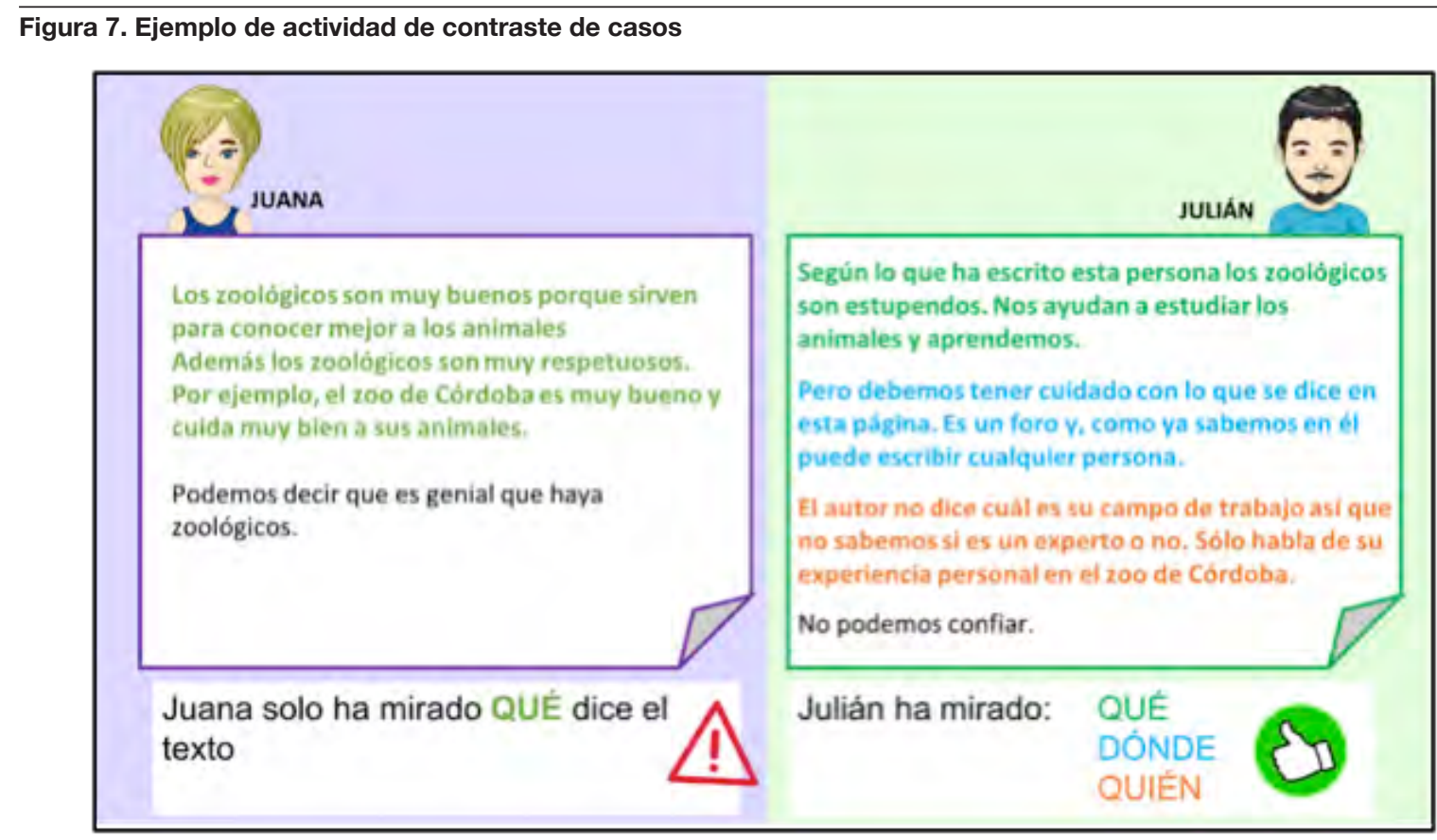

Fuente: elaboración propia.

- Práctica guiada: junto con el modelado y el contraste de casos, el instructor guía a los estudiantes realizando preguntas, recogiendo sus respuestas en los carteles de registro, recapitulando, aclarando los contenidos, señalando los argumentos aportados por los personajes de los distintos módulos al evaluar la fiabilidad de la información encontrada y ofreciendo una reflexión final en cada módulo.

- Discusión grupal: durante cada una de las sesiones instruccionales, los estudiantes han de decidir, mediante las tarjetas de votación y a mano alzada, en qué web confían de entre las páginas web presentadas y posteriormente deben argumentar oralmente dicha decisión. Para ello han de ofrecer al menos una razón en la que se apoyan, las cuales son recogidas por el instructor en los carteles de registro y son utilizadas como base para la discusión grupal, en la que se trata de confrontar los argumentos a favor de una u otra decisión.

\subsection{Procedimiento de las sesiones de intervención}

El programa ha sido diseñado para ser aplicado en grupos pequeños, de entre cinco y diez participantes. La frecuencia de aplicación de las sesiones puede adaptarse a las necesidades de los participantes, aunque consideramos ideal una frecuencia diaria o de días alternos. 


\subsection{Evaluación del aprendizaje}

Un programa de intervención ha de ser evaluado, tanto para conocer el nivel de aprendizaje de los estudiantes como para poder realizar los ajustes necesarios que faciliten un mejor desarrollo del mismo. Para ello, se han implementado distintas técnicas que permiten tanto la evaluación del aprendizaje final como del propio proceso de intervención.

Las sesiones de evaluación pre-test y pos-test siguen el mismo procedimiento. Como ya se ha mencionado anteriormente, se presenta a los participantes una situación en la que un personaje necesita ayuda para decidir qué página de dos posibles es más apropiada para conocer información acerca de un tema controvertido. De manera similar a las sesiones de formación, la información de las páginas web es leída en voz alta (aunque en este caso recomendamos que sea leída por el instructor), tanto el contenido de la información, como la compañía o institución a la que pertenece cada web y la información profesional del autor del texto. Tras finalizar la lectura se aclaran dudas sobre aquellas palabras que los participantes puedan desconocer y se asegura la comprensión del contenido. Los textos usados en ambas sesiones son similares en cuanto al número de palabras y a la clase de referencias a otras fuentes de información insertadas en los textos (ejemplo: referencias a la Organización Mundial de la Salud).

En suma, en estas sesiones se presentan al mismo tiempo dos contenidos web, uno de los cuales es fiable según los objetivos de aprendizaje del programa, mientras que el otro no. Como indicadores de aprendizaje, cada participante debe indicar qué página web considera fiable mediante una tarjeta de votación que todos levantan al mismo tiempo. Posteriormente y por turnos, cada participante ha de justificar oralmente su decisión. Estas sesiones (así como las sesiones de formación) pueden ser grabadas mediante una cámara de vídeo para su posterior análisis. Las justificaciones de cada participante pueden ser así codificadas dependiendo de si se basan en referencias a experiencias personales o conocimientos previos, al contenido de los textos o a los criterios relacionados con la fiabilidad de las fuentes de información: intención de la web o el autor, campo profesional del autor, y el carácter de la web en relación al control de aquello que en ella se publica.

Adicionalmente, puede realizarse una evaluación del aprendizaje a corto plazo. Con un procedimiento similar a las sesiones pre-test y post-test, aunque en esta ocasión de forma individual, cada participante lee por sí mismo dos páginas web presentadas en un ordenador (cuya comprensión ha de ser asegurada por el evaluador) y similarmente debe decidir en cuál confía, justificando su decisión oralmente. Estas sesiones individuales tienen una duración aproximada de 10 minutos.

Además, los participantes realizan en cada una de las sesiones de evaluación dos tareas de recuerdo de las fuentes de información, tomadas como indicadores del grado de atención prestada a dichas fuentes y su procesamiento durante la toma de decisiones. En la primera de las tareas (ver Figura 8), los participantes deben señalar de entre una serie de fuentes posibles aquellas que han aparecido durante las sesiones de evaluación. En la segunda, se presenta a los alumnos una lista de argumentos breves que deben enlazar mediante flechas con las páginas web en las que han aparecido o bien indicar que no han aparecido en ninguna de ellas (ver Figura 9). 
Figura 8. Ejemplo de actividad de recuerdo de fuentes de información

Señala las palabras que aparecían en las páginas que acabáis de leer:

\begin{tabular}{|c|c|c|c|}
\hline Químico & Dentista & $\begin{array}{c}\text { Asociación de } \\
\text { pediatría }\end{array}$ & $\begin{array}{c}\text { Ministerio de } \\
\text { sanidad }\end{array}$ \\
\hline $\begin{array}{c}\text { Asociación de } \\
\text { Consumidores }\end{array}$ & $\begin{array}{c}\text { Hospital Torralva } \\
\text { Salud }\end{array}$ & Enfermera & $\begin{array}{c}\text { Universidad } \\
\text { de París }\end{array}$ \\
\hline $\begin{array}{c}\text { Dentífrico Blanco } \\
\text { Polar }\end{array}$ & $\begin{array}{c}\text { Médicos sin } \\
\text { fronteras }\end{array}$ & $\begin{array}{c}\text { Dentífrico } \\
\text { LimpiaTotal }\end{array}$ & Farmacéutico \\
\hline
\end{tabular}

Fuente: elaboración propia.

Figura 9. Ejemplo de actividad de unión de contenido y fuente de información

Une con una flecha cada frase con la pảgina web en la que aparecia.

Si la frase no aparecia en ninguna web, únelo con "no lo dice ninguna păgina".

\begin{tabular}{|l|}
\hline Es mejor cepillarse sin pasta de dientes. \\
\hline Los cepillos de dientes blandos son mejores. \\
\hline Hay que ir al dentista una vez al año. \\
\hline Las pastas caseras para lavarse los dientes son peligrosas. \\
\hline El chocolate es malo para las caries. \\
\hline La pasta de dientes blanquea los dientes. \\
\hline Las pastas de dientes tienen muy poco flúor. \\
\hline
\end{tabular}

\section{DENTIRIFCO \\ LimpiaTotal}

Hospital IOMnedr Salud

No lo dice ninguna página

Fuente: elaboración propia. 


\section{Conclusiones}

El programa de intervención que presentamos ha demostrado su utilidad para aumentar la atención dedicada a las fuentes de información en internet y para ayudar a jóvenes con DI a evaluar la fiabilidad de la información en internet con base en las características de su fuente (Delgado et al., en prensa). Por ello, creemos que representa una herramienta única para el desarrollo de la lectura crítica en internet en esta población. Por otro lado, nos gustaría subrayar que el diseño del programa facilita su flexibilidad. Aunque dedicado a jóvenes con DI, creemos que representa asimismo una buena herramienta educativa para alumnos de cuarto o quinto de Educación Primaria, y que incluso puede ser aplicado en cursos superiores, siempre que los materiales de trabajo sean adaptados a su nivel educativo, utilizando, por ejemplo, textos más complejos o la lectura individual en lugar de una lectura grupal en voz alta. Asimismo, el programa se puede combinar con otras intervenciones para la enseñanza de habilidades adicionales de la competencia digital, como la búsqueda y lectura de información en internet (Salmerón et al., 2015). Poniendo a disposición de los profesionales el programa pretendemos no sólo estimular la formación de las personas con DI, sino también permitir que la práctica educativa ayude a la mejora del mismo. 


\section{Referencias bibliográficas}

Bråten, l. et al. (2018): "The role of sourcing in discourse comprehension", en Schober, M. et al. (eds.): Handbook of Discourse Processes. Nueva York: Taylor \& Francis.

Chadwick, D. et al. (2013): "Internet access by people with intellectual disabilities: Inequalities and opportunities". Future internet, 5: 376-397 (en línea). <https://doi.org/10.3390/fi5030376>, acceso 13 de diciembre de 2018.

Chiner, E. et al. (2017): "internet use, risks and online behaviour: The view of internet users with intellectual disabilities and their caregivers". British Journal of Learning Disabilities, 45: 190-197 (en línea). <https://doi. org/10.1111/bld.12192>, acceso 13 de diciembre de 2018.

Copeland, S. R. y Keefe, E. B. (2017): "Teaching reading and literacy skills to students with intellectual disability", en Wehmeyer M. L. y Shogren, K. A. (eds.): Handbook of research-based practices for educating students with intellectual disability. Nueva York: Taylor \& Francis.

Delgado, P. et al. (en prensa): "Training young adults with intellectual disability to read critically on the internet". Journal of Applied Research in Intellectual Disabilities.

Fajardo, I. et al. (2016): "Training students with intellectual disabilities to read critically on the internet". Póster presentado en Workshop on Multiple Document Literacy. Universidad de Paris 8, Francia.

Fajardo, I. et al. (2014): "Easy-to-read texts for students with intellectual disability: Linguistic Factors affecting comprehension". Journal of Applied Research in Intellectual Disabilities, 27: 212-25 (en línea). <https://doi. org/10.1111/jar.12065>, acceso 13 de diciembre de 2018.

Greenspan, S. et al. (2001): "Credulity and gullibility in people with developmental disorders: A framework for future research”. International Review of Research in Mental Retardation, 24: 101-135 (en línea). <https://doi. org/10.1016/S0074-7750(01)80007-0>, acceso 13 de diciembre de 2018.

Hetzroni, O. E. y Banin, I. (2017): "The effect of educational software, video modelling and group discussion on social-skill acquisition among students with mild intellectual disabilities". Journal of Applied Research in Intellectual Disabilities, 30: 757-773 (en línea). <https://doi.org/10.1111/jar.12271>, acceso 13 de diciembre de 2018.

Lundberg, I. y Reichenberg, M. (2013): "Developing reading comprehension among students with mild intellectual disabilities: An intervention study". Scandinavian Journal of Educational Research, 57: 89-100 (en línea). <https://doi.org/10.1080/00313831.2011.623179>, acceso 13 de diciembre de 2018.

Mitchell, K. J. et al. (2011): "Youth internet victimization in a broader victimization context". Journal of Adolescent Health, 48: 128-134 (en línea). <https://doi.org/10.1016/j.jadohealth.2010.06.009>, acceso 13 de diciembre de 2018.

Nomura, M. et al. (2010): Guidelines for easy-to-read materials. The Hague: IFLA Headquarters (en línea). <https:// www.ifla.org/files/assets/hq/publications/professional-report/120.pdf>, acceso 13 de diciembre de 2018.

Salmerón, L. et al. (en prensa): "Selection and evaluation of internet information by adults with intellectual disabilities". European Journal of Special Needs Education.

Salmerón, L. et al. (2016): "How students with intellectual disabilities evaluate recommendations from internet forums". Reading and Writing, 29: 1653-1675 (en línea). <https://doi.org/10.1007/s11145-016-9621-4>, acceso 13 de diciembre de 2018. 
Salmerón, L. et al. (2015): “Instrucción de estrategias de lectura digital mediante modelado por video". Informació Psicològica, 110: 38-50 (en línea). <https://doi.org/10.14635/IPSIC.2015.110.5>, acceso 13 de diciembre de 2018.

Salmerón, L. (2013): “Actividades que promueven la transferencia de los aprendizajes: una revisión de la literatura”. Revista de Educación, volumen extraordinario 2013: 34-53 (en línea). <https://doi.org/10.4438/1988-592XRE-2013-EXT-253>, acceso 13 de diciembre de 2018.

Stadtler, M. et al. (2016): “Improving vocational students' consideration of source information when deciding about science controversies". Reading and Writing, 29: 705-729 (en línea). <https://doi.org/10.1007/s11145-0169623-2>, acceso 13 de diciembre de 2018.

Vuorikari, R. et al. (2016): DigComp 2.0: The Digital Competence Framework for Citizens. Update Phase 1: The Conceptual Reference Mod. Publications Office of the European Union (en línea). <https://doi.org/10.2791/11517>, acceso 13 de diciembre de 2018.

Wehmeyer, M. L. (1996): "Self-determination as an educational outcome", en Sands, D. J. y Wehmeyer, M. L. (eds.): Self-determination across the life span. Independence and choice for people with disabilities. Londres: Paul H. Brookes Publishing Co.

Zarcero, S. (2015). "El Club de Lectura Fácil de AFANIAS y su impacto en Pozuelo de Alarcón”. Póster presentado en IX Jornadas Científicas Internacionales de Investigación sobre personas con discapacidad. INICO, Salamanca (en línea). <http://inico.usal.es/cdjornadas2015/CD Jornadas INICO/cdjornadas-inico.usal.es/docs/202. pdf $>$, acceso 13 de diciembre de 2018. 